\title{
ON THE DERIVATIONS IN MAXIMAL ORDERS OF SIMPLE ALGEBRAS
}

\author{
YÛSAKU KAWAHARA
}

1. The theory of derivations and differents in the algebraic number fields or Dedekind rings has been developped by A. Weil [1], Y. Kawada [2] and M. Moriya [4]. Further Y. Kawada [3] has investigated the derivations in maximal orders of simple algebras over number fields. This note is concerned with the derivations in the simple algebras over fields which are quotient fields of $D e$ dekind rings; the commutative rings in which the fundamental theorem of multiplicative ideal theory holds. The auther gives his hearty thanks to Prof. M. Moriya who gave him valuable remarks.

Let $\Re$ be a ring and let $\mathfrak{M}$ be a two-sided $\Re$-module. By a derivation $D$ of $\mathfrak{R}$ into $\mathfrak{M}$, is meant a mapping $D$ of $\mathfrak{R}$ into $\mathfrak{M}$ which satisfies

$$
\begin{aligned}
& D(\alpha+\beta)=D(\alpha)+D(\beta), \text { for } \alpha, \beta \in \Re, \\
& D(\alpha \cdot \beta)=\alpha \cdot D(\beta)+D(\alpha) \cdot \beta .
\end{aligned}
$$

A derivation $D$ is called inner, if there is some element $t$ in $\mathfrak{M}$ such that

$$
D(\alpha)=t \cdot \alpha-\alpha \cdot t
$$

for each element $\alpha$ in $\mathfrak{R}$. The set of all derivations of $\mathfrak{R}$ into $\mathfrak{M}$ constitutes a module $\mathfrak{D}(\mathfrak{R} ; \mathfrak{M})$, and the set of all inner derivations constitutes a submodule $\mathfrak{S}(\Re ; \mathfrak{M})$ of $\mathfrak{D}(\mathfrak{R} ; \mathfrak{M})$. The 1-dimensional cohomology group of $\Re$ for the twosided $\mathfrak{R}$-module $\mathfrak{M}$, denoted $H^{1}(\mathfrak{R} ; \mathfrak{M})$, is the factor module of $\mathfrak{D}(\mathfrak{R} ; \mathfrak{M})$ modulo the submodule of inner derivations. Let $\mathfrak{R}^{\prime}$ be a subring of $\mathfrak{R}$. The set of all derivations $D$ of $\Re$ into $\mathfrak{M}$ such that $D\left(\alpha^{\prime}\right)=0$ for each $\alpha^{\prime}$ in $\mathfrak{R}^{\prime}$ is denoted by $\mathfrak{D}\left(\mathfrak{R}, \mathfrak{R}^{\prime \prime} ; \mathfrak{M}\right)$, and $H^{1}\left(\mathfrak{R}, \mathfrak{R}^{\prime} ; \mathfrak{M}\right)$ is the factor module of $\mathfrak{D}\left(\mathfrak{R}, \mathfrak{R}^{\prime} ; \mathfrak{M}\right)$ modulo $\mathfrak{D}\left(\mathfrak{R}, \mathfrak{R}^{\prime} ; \mathfrak{M}\right) \cap \mathfrak{T}(\mathfrak{R} ; \mathfrak{M})$. Let $\mathfrak{R}^{\prime \prime}$ be a subring of $\mathfrak{R}$ such that if $\alpha^{\prime \prime} \in \mathfrak{H}^{\prime \prime}$, then $\alpha^{\prime \prime} \cdot t=t \cdot \alpha^{\prime \prime}$ for any element $t$ in $\mathfrak{M}$.

Then obviously $\mathfrak{D}\left(\mathfrak{R}, \mathfrak{R}^{\prime} ; \mathfrak{M}\right)$ and $H^{1}\left(\mathfrak{R}, \mathfrak{R}^{\prime} ; \mathfrak{M}\right)$ are considered as $\mathfrak{R}^{\prime \prime}-$ module.

Received April 4, 1955. 
2. Let $k$ be a field which is complete with respect to a discrete valuation. Let $K$ be a finite algebraic extension of $k$ and let $S=\left(\pi_{0}, Z, \sigma\right)$ be a cyclic division algebra over $K$, whose center is $K$, where $Z$ is a cyclic inertial extension of degree $n$ over $K$ (unramified and the residue class field of $Z$ is separable over the residue class field of $K$ ). Further we assume the residue class field of $K$ is separable over the residue class field of $k . \quad \pi_{0}$ is a prime element in $K$ and $\sigma$ is a generating element of the Galois group of $Z / K ; \pi^{n}$ $=\pi_{0}, \pi^{-1} \alpha \pi=\alpha^{\sigma},(\alpha \in Z)$, where $\pi$ is a prime element in $S$. Let $\mathfrak{D}_{S}, \mathfrak{D}_{Z}, \mathfrak{D}_{K}$ and $\mathfrak{D}_{k}$ be the valuation rings of $S, Z, K$ and $k$ respectively, and let $\mathfrak{P}_{s}, \mathfrak{P}_{K}$ be the prime ideals in $\mathfrak{D}_{S}$ and $\mathfrak{D}_{K}$.

$$
\mathfrak{D}_{s}=\mathfrak{D}_{z}+\pi \mathfrak{D}_{z}+\ldots+\pi^{n-1} \mathfrak{D}_{z} .
$$

Let $\left(1, \omega, \omega^{2}, \ldots, \omega^{n-1}\right)$ be a base of $\mathfrak{D}_{Z}$ with respect to $\mathfrak{D}_{K}$. Now $\mathfrak{D}_{S} / \mathfrak{P}_{S}^{r}$ is considered as two-sided $\mathfrak{D}_{s}$-module.

Lemma 1. Let $D \in \mathscr{D}\left(\mathfrak{D}_{s}, \mathfrak{D}_{k} ; \mathfrak{D}_{s} / \mathfrak{P}_{s}^{r}\right)$ and $D(\omega) \equiv \alpha\left(\mathfrak{P}_{s}^{r}\right) \alpha \in \mathfrak{D}_{z}$. Then the restriction of $D$ on $\mathfrak{D}_{z}$ is a derivation of $\mathfrak{D}_{z}$ into $\mathfrak{D}_{z} / \mathfrak{D}_{Z} \cap \mathfrak{P}_{s}^{r}$ and there is an element $\xi$ in $\mathfrak{D}_{z}$ such that $D(\pi) \equiv \pi \xi, \bmod \mathfrak{P}_{s}^{r}$.

Proof. Let $\beta$ be an element in $\mathfrak{D}_{z}$. Then $\omega \beta=\beta \omega$. Therefore

Put

$$
\begin{gathered}
\omega D(\beta)+D(\omega) \beta \equiv \beta D(\omega)+D(\beta) \omega, \\
\omega D(\beta)+\alpha \beta \equiv \beta \alpha+D(\beta) \omega, \text { hence } \\
D(\beta) \omega-\omega D(\beta) \equiv 0 \quad \bmod \mathfrak{P}_{s}^{r} .
\end{gathered}
$$

Then

$$
D(\beta) \equiv \eta_{0}+\pi \eta_{1}+\ldots+\pi^{n-1} \eta_{n-1} \quad\left(\mathfrak{P}_{S}^{r}\right) .
$$

$$
\pi \eta_{1}\left(\omega-\omega^{\sigma}\right)+\ldots+\pi^{n-1} \eta_{n-1}\left(\omega-\omega^{\sigma^{n-1}}\right) \equiv 0 \quad\left(\mathfrak{P}_{s}^{r}\right) .
$$

Since $\omega-\omega^{\sigma^{i}}$ is not divisible by $\mathfrak{P}_{S}$ for $1 \leqq i \leqq n-1$, we get

Therefore

$$
\pi \eta_{1} \equiv 0, \ldots, \pi^{n-1} \eta_{n-1} \equiv 0 \quad\left(\Re_{s}^{r}\right) .
$$

Now, let

$$
D(\beta) \equiv \eta_{0} \quad \bmod \mathfrak{P}_{s}^{r}
$$

Then since

$$
D(\pi) \equiv \xi_{0}+\pi \xi_{1}+\ldots+\pi^{n-1} \xi_{n-1} \quad \bmod \mathfrak{P}_{s}^{r} .
$$

$$
\begin{gathered}
D(\pi) \omega^{\sigma}+\pi D\left(\omega^{\sigma}\right) \equiv \omega D(\pi)+D(\omega) \pi \quad \bmod \mathfrak{H}_{s}^{r}, \\
\xi_{0} \omega^{\sigma}+\pi \xi_{1} \omega^{\sigma}+\ldots+\pi^{n-1} \xi_{n-1} \omega^{\sigma}-\left(\omega \xi_{0}+\omega \pi \xi_{1}\right. \\
\left.+\ldots+\omega \pi^{n-1} \xi_{n-1}\right)+\pi\left(D\left(\omega^{\sigma}\right)-D(\omega)\right) \equiv 0
\end{gathered}
$$




$$
\begin{gathered}
\xi_{0}\left(\omega-\omega^{\sigma}\right)+\pi^{2} \xi_{2}\left(\omega^{\sigma}-\omega^{\sigma^{2}}\right)+\ldots+\pi^{n-1} \xi_{n-1}\left(\omega^{\sigma}-\omega^{\sigma^{n-1}}\right) \\
+\pi\left(D\left(\omega^{\sigma}\right)-D(\omega)\right) \equiv 0 \quad\left(\mathfrak{F}_{S}^{r}\right) .
\end{gathered}
$$

Therefore, we get

hence

$$
\xi_{0} \equiv 0 \quad \pi^{2} \xi_{2} \equiv 0, \ldots, \pi^{n-1} \xi_{n=1} \equiv 0 \quad\left(\mathfrak{P}_{s}^{r}\right),
$$

$$
D(\pi) \equiv \pi \xi 1 \quad \bmod \mathfrak{P}_{s}^{r}
$$

Lemma 2. Let $D \in \mathscr{D}\left(\mathfrak{D}_{s}, \mathfrak{D}_{k} ; \mathfrak{D}_{s} / \mathfrak{P}_{s}^{r}\right)$ and $D(\omega) \equiv \alpha\left(\mathfrak{P}_{s}^{r}\right), \alpha \in \mathfrak{D}_{z}$. Then the restriction of $D$ on $\mathfrak{D}_{K}$ is a derivation of $\mathfrak{D}_{K}$ into $\mathfrak{D}_{K} / \mathfrak{D}_{K} \cap \mathfrak{P}_{s}^{r}$, and

$$
\pi_{0} S p_{Z / K}(\xi) \equiv D\left(\pi_{0}\right) \quad \bmod \mathfrak{P}_{s}^{r},
$$

where $D(\pi) \equiv \pi \xi \bmod \mathfrak{P}_{s}^{r}$.

Proof. Since $\pi^{n}=\pi$,

$$
\begin{aligned}
D\left(\pi_{0}\right) & =D\left(\pi^{n}\right)=\sum_{i+j=n-1} \pi^{i} D(\pi) \pi^{j} \equiv \sum_{i+j=n-1} \pi^{i} \pi \xi \pi^{j} \\
& =\sum_{i+j=n-1} \pi^{i} \pi \pi^{j} \xi^{\sigma^{j}}=\sum_{j=0}^{n-1} \pi^{n} \xi^{\sigma^{j}}=\pi_{0} S p_{Z / K}(\xi) \quad \bmod \mathfrak{P}_{s}^{r} .
\end{aligned}
$$

As $\pi_{0} S p(\xi) \in \mathfrak{D}_{K}, D\left(\pi_{0}\right) \in \mathfrak{D}_{K} / \mathfrak{Q}_{K} \cap \mathfrak{P}_{s}^{r}$.

Now, since the residue class field of $K$ is separable over the residue class field of $k$, there is a subfield $K^{*}$ of $K$ such that $K^{*}$ is unramified over $k$ and the residue class field of $K^{*}$ coincides with that of $K$ over the residue class field of $k$. Let $\mathfrak{D}^{*}$ be the valuation ring of $K^{*}$ and let $\omega_{1}, \ldots, \omega_{s}$ be a base of $\mathfrak{D}^{*}$ over $\mathfrak{D}_{k}$. Then, as $K^{*}$ is unramified over $k, \mathfrak{D}\left(\mathfrak{D}^{*}, \mathfrak{D}_{k} ; \mathfrak{D}_{z} / \mathfrak{D}_{z} \cap \mathfrak{P}_{S}^{r}\right)$ $=\{0\}^{1{ }^{1}} \quad$ Therefore $D\left(\omega_{i}\right) \equiv 0 \bmod \mathfrak{P}_{s}^{r} ;$ moreover since $\pi_{0}^{i} \omega_{j}(j=1, \ldots, s$, $i=0,1, \ldots)$ is a base of $\mathfrak{D}_{K}$ over $\mathfrak{D}_{k}$, we see that for any element $\beta$ in $\mathfrak{D}_{K}$, $D(\beta)$ is congruent to an element in $\mathfrak{D}_{K} \bmod \mathfrak{P}_{s}^{r}$. This shows that the restriction of $D$ on $\mathfrak{D}_{K}$ is a derivation of $\mathfrak{D}_{K}$ into $\mathfrak{D}_{K} / \mathfrak{D}_{K} \cap \mathfrak{P}_{S}^{r}$.

Lemma 3. Let $D^{\prime \prime}$ be a derivation of $\mathfrak{D}_{z}$ into $\mathfrak{D}_{z} / \mathfrak{D}_{z} \cap \mathfrak{P}_{s}^{r}$, which is an extension of a derivation $D^{\prime}$ of $\mathfrak{D}_{K}$ into $\mathfrak{D}_{K} / \mathfrak{D}_{K} \cap \mathfrak{P}_{s}^{r}$. Then for $\eta \in \mathfrak{D}_{Z}$ and for any automorphism $\tau$ of $Z / K, D^{\prime \prime}\left(\eta^{\tau}\right) \equiv D^{\prime \prime}(\eta)^{\tau} \bmod \mathfrak{P}_{s}^{r}$.

Proof. Put $\bar{D}(\eta) \equiv D^{\prime \prime}\left(\eta^{\tau}\right)^{\tau-1} \bmod \mathfrak{P}_{s}^{r}$. Then $\bar{D}$ is a derivation of $\mathfrak{D}_{z}$ into $\mathfrak{D}_{Z} / \mathfrak{D}_{z} \cap \mathfrak{P}_{s}^{r}$, which coincides with $D^{\prime \prime}$ on $\mathfrak{D}_{K}$. Since $Z / K$ is unramified, there is only one extension of $D^{\prime} \cdot{ }^{1)}$ Therefore $\bar{D}=D^{\prime \prime}$ and hence $D^{\prime \prime}\left(\eta^{\tau}\right) \equiv D^{\prime \prime}(\eta)^{\tau}$ $\bmod \mathfrak{P}_{s}^{r}$.

1) M. Moriya [4], p. 134, Satz 5 and Satz 6. 
Lemma 4. Let $D^{\prime} \in \mathfrak{D}\left(\mathfrak{D}_{K}, \mathfrak{D}_{k} ; \mathfrak{D}_{K} / \mathfrak{D}_{K} \cap \mathfrak{P}_{S}^{r}\right)$. If $\xi \in \mathfrak{D}_{Z}$ and $\pi_{0} \operatorname{Sp}_{Z / K}(\xi)$ $\equiv D^{\prime}\left(\pi_{0}\right) \bmod \mathfrak{P}_{s}^{r}$, then there is a derivation $D$ in $\mathfrak{D}\left(\mathfrak{D}_{s}, \mathfrak{D}_{k} ; \mathfrak{D}_{s} / \mathfrak{P}_{s}^{r}\right)$ which is an extension of $D^{\prime}$, satisfying $D(\pi) \equiv \pi \xi \bmod \mathfrak{P}_{s}^{r}$.

Proof. There is a uniquely determined derivation $D^{\prime \prime}$ of $\mathfrak{D}_{z}$ into $\mathfrak{D}_{Z} / \mathfrak{D}_{Z} \cap \mathfrak{H}_{S}^{r}$, such that $D^{\prime \prime}$ is an extension of $D^{\prime}$. We put $D\left(\sum_{i=0}^{n-1} \pi^{i} \eta_{i}\right)=\sum_{i=0}^{n-1} \pi^{i} D^{\prime \prime}\left(\eta_{i}\right)+\sum_{i=0}^{n-1} D\left(\pi^{i}\right) \eta_{i}$, $\eta_{i} \in \mathfrak{D}_{z}$, where

$$
D\left(\pi^{i}\right) \equiv \pi^{i}\left(\xi+\xi^{\circ}+\ldots+\xi^{\circ-1}\right) \quad \bmod \mathfrak{P} \xi_{s}^{r} .
$$

Then for $i+j<n$,

$$
\begin{aligned}
D\left(\pi^{i} \pi^{j}\right) & \equiv \pi^{i+j}\left(\xi+\xi^{\sigma}+\ldots+\xi^{\sigma^{i+j-1}}\right) \\
& \equiv \pi^{i}\left(\xi+\xi^{\sigma}+\ldots+\xi^{\alpha^{i-1}}\right) \cdot \pi^{j}+\pi^{i} \pi^{j}\left(\xi+\xi^{\sigma}+\ldots \xi^{\sigma^{j-1}}\right) \\
& \equiv D\left(\pi^{i}\right) \pi^{j}+\pi^{i} D\left(\pi^{j}\right) \quad \bmod \mathfrak{P}_{S .}^{r} .
\end{aligned}
$$

For $i+j \geqq n, i<n, j<n$,

$$
\begin{aligned}
D\left(\pi^{i} \pi^{j}\right) & =D\left(\pi^{i+j-n} \pi_{0}\right)=\pi^{i+j-n}\left(\xi+\xi^{\sigma}+\ldots+\xi^{\sigma^{i+j-n-1}}\right) \pi_{0}+\pi^{i+j-n} D^{\prime}\left(\pi_{0}\right) \\
& \equiv \pi^{i+j}\left(\xi^{o^{n}}+\xi^{\sigma^{n+1}}+\ldots+\xi^{\sigma^{i+j-1}}\right)+\pi^{i+j} S p_{Z / K}(\xi) \\
& \equiv \pi^{i+j}\left(\xi+\xi^{\sigma}+\ldots+\xi^{\sigma^{i+j-1}}\right) \equiv \pi^{i} D\left(\pi^{j}\right)+D\left(\pi^{i}\right) \pi^{j} .
\end{aligned}
$$

Therefore for $i+j<n$ by using Lemma 3,

$$
\begin{aligned}
& D\left(\pi^{i} \eta_{i} \cdot \pi^{j} \eta_{j}\right)=D\left(\pi^{i} \pi^{j} \eta_{i}^{\gamma^{j}} \eta_{j}\right)=\pi^{i+j} D^{\prime \prime}\left(\eta_{i}^{\tau^{j}} \eta_{j}\right)+D\left(\pi^{i+j}\right) \eta_{i}^{\tau^{i}} \eta_{i j} \\
& =\pi^{i} \pi^{j} D^{\prime \prime}\left(\eta_{i}^{\sigma_{i}^{j}}\right) \eta_{j}+\pi^{i} \pi^{j} \eta_{i}^{\pi^{j}} D^{\prime \prime}\left(\eta_{j}\right)+\pi^{i} D\left(\pi^{j}\right) \eta_{i}^{\pi^{j}} \eta_{j}+D\left(\pi^{i}\right) \pi^{j} \eta_{i}^{{ }^{j}} \eta_{j} \\
& =\left[\pi^{i} D^{\prime \prime}\left(\eta_{i}\right)+D\left(\pi^{i}\right) \eta_{i}\right] \pi^{j} \eta_{j}+\pi^{i} \eta_{i}\left[\pi^{j} D^{\prime \prime}\left(\eta_{j}\right)+D\left(\pi^{j}\right) \eta_{j}\right] \\
& =D\left(\pi^{i} \gamma_{i}\right) \pi^{j} \eta_{j}+\pi^{i} \eta_{i} D\left(\pi^{j} \eta_{j}\right) \text {. }
\end{aligned}
$$

Similarly we get

$$
D\left(\pi^{i} \eta_{i} \pi^{j} \eta_{j}\right)=D\left(\pi^{i} \eta_{i}\right) \pi^{j} \eta_{j}+\pi^{i} \eta_{i} D\left(\pi^{j} \eta_{j}\right) \text { for } i+j \geqq n .
$$

Hence it follows easily that $D$ is a derivation of $\mathfrak{D}_{s}$ into $\mathfrak{D}_{s} / \mathfrak{F}_{s}^{r}$.

Lemma 5. Let $W$ be an unramified Galois extension of $K$ such that the residue class field of $W$ is separable over the residue class field of $K$. If a mapping $\sigma \rightarrow a_{\sigma}$ of the Galois group $\mathbb{B}$ of $W / K$ into $\mathfrak{D}_{W} / \mathfrak{P}_{W}^{r}$ satisfies the conditions:

$$
a_{\circ \tau} \equiv a_{\sigma}^{\tau}+a_{\tau} \quad \text { for all } \sigma, \tau \in \mathbb{B},
$$

then there is an element $b \in \mathfrak{D}_{W}$ such that $a_{\circ} \equiv b-b^{\Im} \bmod \mathfrak{P}_{W^{*}}^{r}$ for all $\sigma$.

Proof. Since the residue class field of $W$ is separable over that of $K$ there is an element $v$ in $\mathfrak{D}_{W}$ such that $S_{W / K}(v) \neq 0 \bmod \mathfrak{P}_{W}$. Put 
Then

$$
b \equiv \frac{1}{S p(v)} \sum_{\sigma \in \mathbb{S}} v^{\sigma} a_{\sigma} \quad \bmod \mathfrak{P}_{W}^{r}
$$

Therefore

$$
\begin{aligned}
b^{\tau} & \equiv \frac{1}{S p(v)} \sum_{\sigma} v^{\sigma \tau} a_{\sigma}^{\tau} \equiv \frac{1}{S p(v)} \sum_{\sigma} v^{\sigma \tau}\left(a_{\sigma \tau}-a_{\tau}\right) \\
& =\frac{1}{S p(v)} \sum_{\sigma} v^{\sigma \tau} a_{\sigma \tau}-a_{\tau} \quad \bmod \mathfrak{P}_{W}^{r} .
\end{aligned}
$$

$$
a_{\tau} \equiv b-b^{\tau} \quad \bmod \mathfrak{P}_{w}^{r} .
$$

Lemma 6. Let $Z$ be a cyclic unramified extension of $K$ such that the residue class field of $Z$ is separable over that of $K$ and let $\sigma$ be a generator of the Galois group \& of $Z / K$. Then, for an element $a$ in $\mathfrak{D}_{Z}$ in order to be

$$
S p_{Z / K}(a) \equiv 0 \quad \bmod \mathfrak{P}_{Z}^{r},
$$

it is necessary and sufficient that there is an element $b$ in $D_{z}$ such that

$$
a \equiv b-b^{3} \quad \bmod \mathfrak{P}_{z}^{r} .
$$

Proof. Put $a_{\sigma} \equiv 1, a_{\sigma} \equiv a, a_{\sigma^{2}} \equiv a+a^{\sigma}, \ldots, a_{\circ} n-1 \equiv a+a^{\sigma}+\ldots a^{\sigma^{n-2}}$, where $n$ denotes the order of $\mathbb{S}$. Then $\sigma^{i} \rightarrow a_{\sigma^{i}}$ satisfies the condition of the preceding lemma. The converse is obvious.

Theorem 1. Let $S=\left(\pi_{0}, Z, \sigma\right)$. Then

$$
H^{1}\left(\mathfrak{D}_{S}, \mathfrak{D}_{K} ; \mathfrak{D}_{S} / \mathfrak{P}_{S}^{r}\right)\left\{\begin{array}{l}
=\{0\} \text { for } r \equiv 1 \quad \bmod n \\
\cong \mathfrak{D}_{K} / \mathfrak{P}_{K} \quad\left(\text { as } \mathfrak{D}_{K} \text {-module }\right) \text { otherwise. }{ }^{2)}
\end{array}\right.
$$

Proof. $\mathfrak{D}_{z}=\mathfrak{D}_{K}\left[1, \omega, \omega^{2}, \ldots, \omega^{n-1}\right]$

Let $D \in \mathfrak{D}\left(\mathfrak{D}_{s}, \mathfrak{D}_{K} ; \mathfrak{D}_{s} / \mathfrak{P}_{s}^{r}\right)$ and let

$$
D(\omega) \equiv \sum_{i=0}^{n-1} \pi^{i} \eta_{i}, \quad \eta_{i} \in \mathfrak{D}_{z} .
$$

Put $\sum_{i=1}^{n-1} \pi^{i} \eta_{i}\left(\omega-\omega^{\sigma^{i}}\right)^{-1}=\gamma$. Then since $\omega-\omega^{\sigma^{i}} \equiv 0\left(\mathfrak{P}_{s}\right)$ for $1 \leqq i \leqq n-1$, we get $\gamma \in \mathfrak{D}_{s}$. Let $D^{\prime}$ be the inner derivation defined by

Then

$$
D^{\prime}(\alpha) \equiv \gamma \cdot \alpha-\alpha \cdot \gamma \quad \bmod \mathfrak{P}_{s}^{r} .
$$

$$
\begin{aligned}
D^{\prime}(\omega) & \equiv \gamma \omega-\omega \gamma \equiv \sum_{i=1}^{n-1} \pi^{i} \eta_{i}\left(\omega-\omega^{\sigma^{i}}\right)^{-1} \omega-\sum_{i=1}^{n-1} \omega \pi^{i} \eta_{i}\left(\omega-\omega^{\sigma^{i}}\right)^{-1} \bmod \mathfrak{P}_{S}^{r} \\
& \equiv \sum_{i=1}^{n-1} \pi^{i}\left[\eta_{i}\left(\omega-\omega^{\sigma^{i}}\right)^{-1} \omega-\omega^{\sigma^{i}} \eta_{i}\left(\omega-\omega^{\sigma^{i}}\right)^{-1}\right] \equiv \sum_{i=1}^{n-1} \pi^{i} \eta_{i} .
\end{aligned}
$$

2) Y. Kawada [3], Theorem 1. 
Moreover $D^{\prime} \in \mathscr{D}\left(\mathfrak{D}_{s}, \mathfrak{D}_{K} ; \mathfrak{D}_{s} / \mathfrak{P}_{s}^{r}\right)$. We set $D^{\prime \prime}=D-D^{\prime}$, then $D^{\prime \prime}(\omega) \equiv \eta_{0}$ $\bmod \mathfrak{P}_{s}^{r}, \eta_{0} \in \mathfrak{D}_{z}$. Hence the restriction of $D^{\prime \prime}$ on $\mathfrak{D}_{z}$ is a derivation of $\mathfrak{D}_{z}$ into $\mathfrak{O}_{z} / \mathfrak{D}_{z} \cap \mathfrak{P}_{s}^{r}$, moreover clearly the restriction of $D^{\prime \prime}$ belongs to $\mathfrak{D}\left(\mathfrak{D}_{z}, \mathfrak{D}_{K}\right.$; $\left.\mathfrak{D}_{Z} / \mathfrak{D}_{Z} \cap \mathfrak{P}_{s}^{r}\right)$. Therefore, since $Z / K$ is unramified we get $D^{\prime \prime}(\alpha) \equiv 0$ for any $\alpha \in \mathfrak{D}_{z}{ }^{3)}$ which shows that

$$
\begin{aligned}
H^{1}\left(\mathfrak{D}_{s}, \mathfrak{D}_{K} ; \mathfrak{D}_{s} / \mathfrak{P}_{s}^{r}\right) & \cong \mathfrak{D}\left(\mathfrak{D}_{s}, \mathfrak{D}_{z} ; \mathfrak{D}_{s} / \mathfrak{P}_{s}^{r}\right) / \mathfrak{D}\left(\mathfrak{D}_{s}, \mathfrak{D}_{z} ; \mathfrak{D}_{s} / \mathfrak{P}_{s}^{r}\right) \cap \\
& \cap \mathfrak{S}\left(\mathfrak{D}_{s}, \mathfrak{D}_{K} ; \mathfrak{D}_{s} / \mathfrak{P}_{s}^{r}\right) .
\end{aligned}
$$

By Lemma 2 and Lemma 4 , there is a derivation $D$ in $\mathscr{D}\left(\mathfrak{D}_{s}, \mathfrak{D}_{z} ; \mathfrak{D}_{s} / \mathfrak{F}_{s}^{r}\right)$ satisfying

if and only if

$$
D(\pi) \equiv \lambda \quad \bmod \mathfrak{P}_{S}^{r}
$$

$$
\begin{aligned}
& \lambda \equiv \pi \xi, \quad \xi \in \mathfrak{D}_{z}, \quad \text { where } \\
& \pi_{0} S p_{Z / K} \xi \equiv 0 \quad \bmod \mathfrak{P}_{s}^{r},
\end{aligned}
$$

and moreover in this case $D \in \mathcal{S}\left(\mathfrak{Q}_{S}, \mathfrak{D}_{K} ; \mathfrak{D}_{S} / \mathfrak{P}_{S}^{r}\right)$ if and only if $S p_{Z / K}(\xi) \equiv 0$ $\bmod \mathfrak{P}_{s}^{r-1}$, for if $S p_{Z / K}(\xi) \equiv 0\left(\mathfrak{P}_{s}^{r-1}\right)$, in virtue of Lemma 6 , there is an element $\eta$ in $\mathfrak{D}_{z}$ such that $\xi \equiv \eta-\eta^{\sigma} \bmod \mathfrak{P}_{s}^{r-1}$, hence $-(\eta \pi-\pi \eta)=\pi\left(\eta-\eta^{\sigma}\right) \equiv \pi \xi \bmod$ $\mathfrak{P}_{s}^{r}$; conversely if $D$ is inner defined by $D(\alpha) \equiv \gamma \alpha-\alpha \gamma, \gamma \equiv \eta+\pi \eta_{1}+\ldots$ $+\pi^{n-1} \eta_{n-1}$, then since $D \in \mathscr{D}\left(\mathfrak{D}_{s}, \mathfrak{D}_{z} ; \mathfrak{D}_{S} / \mathfrak{P}_{s}^{r}\right), \quad D(\omega) \equiv \gamma \omega-\omega \gamma \equiv \pi \eta_{1}\left(\omega-\omega^{\circ}\right)$ $+\ldots+\pi^{n-1} \eta_{n-1}\left(\omega-\omega^{\sigma^{n-1}}\right) \equiv 0 \bmod \mathfrak{P}_{s}^{r}$, therefore we get $\eta \equiv \gamma \bmod \mathfrak{P}_{s}^{r}$ and $D(\pi) \equiv \pi \xi \equiv \pi\left(\eta-\eta^{\natural}\right) \bmod \mathfrak{P}_{s}^{r}$, which concludes that $S p_{Z / K} \xi \equiv 0 \bmod \mathfrak{P}_{S}^{r-1}$. Further as the residue class field of $Z$ is separable over that of $K$, we have $S p_{Z / K}\left(\mathfrak{D}_{Z}\right)=\mathfrak{D}_{K}$. Therefore the mapping $D \rightarrow S p_{Z / K}(\xi)$ induces the isomorphism between $\mathfrak{D}\left(\mathfrak{D}_{s}, \mathfrak{D}_{z} ; \mathfrak{D}_{s} / \mathfrak{P}_{S}^{r}\right) / \mathfrak{D}\left(\mathfrak{D}_{S}, \mathfrak{D}_{z} ; \mathfrak{D}_{S} / \mathfrak{P}_{S}^{r}\right) \cap \mathfrak{S}\left(\mathfrak{D}_{s}, \mathfrak{D}_{K} ; \mathfrak{D}_{s} / \mathfrak{P}_{s}^{r}\right)$ and the module of all the elements $a \bmod \mathfrak{P}_{s}^{r-1}, a \in \mathfrak{D}_{K}$ satisfying $\pi_{0} a \equiv 0 \bmod \mathfrak{P}_{s}^{r}$. From this we get our theorem.

THEOREM 2. $H^{1}\left(\mathfrak{D}_{S}, \mathfrak{D}_{k} ; \mathfrak{D}_{S} / \mathfrak{F}_{S}^{r}\right) / H^{1}\left(\mathfrak{D}_{S}, \mathfrak{D}_{K} ; \mathfrak{D}_{S} / \mathfrak{P}_{S}^{r}\right) \cong H^{1}\left(\mathfrak{D}_{K}, \mathfrak{D}_{k} ; \mathfrak{D}_{K} / \mathfrak{D}_{K}\right.$ $\left.\cap \mathfrak{P}_{s}^{r}\right)=\mathfrak{D}\left(\mathfrak{D}_{K}, \mathfrak{D}_{k} ; \mathfrak{D}_{K} / \mathfrak{O}_{K} \cap \mathfrak{P}_{s}^{r}\right)$ (as $\mathfrak{D}_{K^{-}}$module) for $r$ such that $\mathfrak{P}_{K}^{d} \mid \mathfrak{F}_{s}^{r-1}$, where $\mathfrak{P}_{K}^{d}$ denotes the different of $K$ with respect to $k$.

Proof. As in the proof of Theorem 1 every class of $H^{1}\left(\mathfrak{D}_{s}, \mathfrak{D}_{k} ; \mathfrak{D}_{s} / \mathfrak{P}_{s}^{r}\right)$ contains a derivation $D$ such that $D(\omega) \equiv \alpha \bmod \mathfrak{P}_{s}^{r}, \alpha \in \mathfrak{D}_{z} . \quad$ By Lemma 2 the restriction of $D$ on $\mathfrak{D}_{K}$ is a derivation $D^{\prime}$ of $\mathfrak{D}_{K}$ into $\mathfrak{D}_{K} / \mathfrak{D}_{K} \cap \mathfrak{P}_{s}^{r}$. Moreover if $D$ is inner, $D^{\prime}$ is zero derivation. Therefore it is easily seen that the mapping

\footnotetext{
3) M. Moriya [4], Satz 5.
} 
$D \rightarrow D^{\prime}$ is a homomorphism of $H^{1}\left(\mathfrak{D}_{s}, \mathfrak{D}_{k} ; \mathfrak{D}_{s} / \mathfrak{P}_{s}^{r}\right)$ into $\mathfrak{D}\left(\mathfrak{D}_{K}, \mathfrak{D}_{k} ; \mathfrak{D}_{K} / \mathfrak{D}_{K}\right.$ $\left.\cap \mathfrak{F}_{S}^{r}\right)$, and obviously its kernel is $H^{1}\left(\mathfrak{D}_{S}, \mathfrak{D}_{K} ; \mathfrak{D}_{S} / \mathfrak{P}_{S}^{r}\right)$, hence $H^{1}\left(\mathfrak{D}_{S}, \mathfrak{D}_{k}\right.$; $\left.\mathfrak{D}_{s} / \mathfrak{P}_{s}^{r}\right) / H^{1}\left(\mathfrak{D}_{S}, \mathfrak{D}_{K} ; \mathfrak{D}_{s} / \mathfrak{P}_{s}^{r}\right)$ is isomorphic to a submodule of $\mathfrak{D}\left(\mathfrak{D}_{K}, \mathfrak{D}_{k} ; \mathfrak{D}_{K} / \mathfrak{D}_{K}\right.$ $\left.\cap \mathfrak{P}_{s}^{r}\right)$.

Now we assume $\mathfrak{P}_{K}^{d} \mid \mathfrak{P}_{s}^{r-1}$. Then if $D^{\prime} \in \mathfrak{D}\left(\mathfrak{D}_{K}, \mathfrak{D}_{k} ; \mathfrak{D}_{K} / \mathfrak{D}_{K} \cap \mathfrak{P}_{s}^{r}\right)$,

$$
D^{\prime}\left(\pi_{0}\right) \equiv 0 \quad\left(\mathfrak{P}_{K}\right)^{4}{ }^{4}
$$

Therefore there is an element $\eta$ in $\mathfrak{D}_{K}$ satisfying

$$
\pi_{0} \eta \equiv D^{\prime}\left(\pi_{0}\right) \quad \bmod \mathfrak{P}_{s}^{r} .
$$

Hence by Lemma 4 there is a derivation $D$ in $\mathscr{D}\left(\mathfrak{D}_{s}, \mathfrak{D}_{k} ; \mathfrak{D}_{s} / \mathfrak{P}_{s}^{r}\right)$ which is an extension of $D^{\prime}$, which shows that

$$
H^{1}\left(\mathfrak{D}_{s}, \mathfrak{D}_{k} ; \mathfrak{D}_{s} / \mathfrak{P}_{s}^{r}\right) / H^{1}\left(\mathfrak{D}_{s}, \mathfrak{D}_{K} ; \mathfrak{D}_{s} / \mathfrak{P}_{s}^{r}\right) \cong \mathfrak{D}\left(\mathfrak{D}_{K}, \mathfrak{D}_{k} ; \mathfrak{D}_{K} / \mathfrak{D}_{K} \cap \mathfrak{P}_{s}^{r}\right) .
$$

Theorem 3. Let $A$ be a full matrix ring in any division algebra $S$ over $k$. Let $\mathfrak{D}_{A}$ be a maximal order of $A$ and $\mathfrak{\beta}_{A}$ the two-sided prime ideal of $\mathfrak{D}_{A}$. Then

$$
H^{1}\left(\mathfrak{D}_{A}, \mathfrak{D}_{k} ; \mathfrak{D}_{A} / \mathfrak{P}_{A}^{r}\right) \cong H^{1}\left(\mathfrak{D}_{s}, \mathfrak{D}_{k} ; \mathfrak{D}_{s} / \mathfrak{P}_{s}^{r}\right)^{5)}
$$

Proof. Let $e_{i j}(i, j=1, \ldots, m)$ denote a system of matrix units of $A$. Then we can assume that $\mathfrak{D}_{A}=\sum_{j, i} e_{i j} \mathfrak{D}_{S}$, and $\mathfrak{P}_{A}=\sum_{i, j} e_{i j} \mathfrak{P}_{s}{ }^{6)}$

Let $D$ be in $\mathscr{D}\left(\mathfrak{D}_{A}, \mathfrak{D}_{k} ; \mathfrak{D}_{A} / \mathfrak{P}_{A}^{r}\right)$ and put

$$
z \equiv \sum_{i=1}^{m} e_{i 1} D\left(e_{1 i}\right) \quad \bmod \mathfrak{P}_{A}^{r} \text {. }
$$

Then

$$
\begin{aligned}
e_{h t} z-z e_{h t} & \equiv \sum_{i=1}^{m} e_{h t} e_{i 1} D\left(e_{1 i}\right)-\sum_{i=1}^{m} e_{i 1} D\left(e_{1 i}\right) e_{h t} \\
& \equiv \sum_{i=1}^{m} e_{h t} e_{i 1} D\left(e_{1 i}\right)-\sum_{i=1}^{m} e_{i 1} D\left(e_{1 i} e_{h t}\right)+\sum_{i=1}^{m} e_{i 1} e_{1 i} D\left(e_{h t}\right) \\
& \equiv D\left(e_{h t}\right) \quad \bmod \mathfrak{P}_{A}^{r} .
\end{aligned}
$$

Let $D^{\prime}$ be an inner derivation defined by

$$
D^{\prime}(a) \equiv a z-z a, \quad a \in D_{A} .
$$

4) Let $T$ be the inertial subfield of $K$ over $k$ and let $f(X)$ be the irreducible polynomial in $T[X]$ such that $f\left(\pi_{0}\right)=0$. Then $f^{\prime}\left(\pi_{0}\right) D_{K^{\prime}}=\mathfrak{P}_{K}^{d}$ and $D^{\prime}(a) \equiv 0\left(\mathfrak{P}_{K}^{r}\right)$ for $a \in D_{T}$. Therefore $f^{\prime}\left(\pi_{0}\right) D^{\prime}\left(\pi_{0}\right) \equiv 0 \bmod \mathfrak{P}_{K}^{r}$ and hence $D^{\prime}\left(\pi_{0}\right) \equiv 0 \bmod \mathfrak{B}_{K}$.

5) Y. Kawada [3], Theorem 2.

6) H. Hasse [5]. 
Then $\left(D-D^{\prime}\right)\left(e_{h t}\right) \equiv 0 \bmod \mathfrak{P}_{A}^{r},(h, t=1, \ldots, m)$ and hence if $a=\sum_{h, t} e_{h t} \alpha_{h t}$, $\alpha_{h t} \in \mathfrak{D}_{s}$,

$$
\left(D-D^{\prime}\right) a \equiv \sum_{h, t} e_{h t} D\left(\alpha_{h t}\right) \quad \bmod \mathfrak{F}_{A}^{r} .
$$

Moreover, for $\alpha \in \mathfrak{D}_{s}$

$$
\begin{aligned}
\left(D-D^{\prime}\right)\left(e_{h t} \alpha\right) & \equiv e_{h t}\left(D-D^{\prime}\right)(\alpha) \\
& \equiv\left(D-D^{\prime}\right)\left(\alpha e_{h t}\right) \equiv\left(D-D^{\prime}\right)(\alpha) e_{h t} \quad \bmod \mathfrak{P}_{A}^{r} .
\end{aligned}
$$

Therefore we must have

$$
\left(D-D^{\prime}\right)(\alpha) \equiv \sum_{i=1}^{m} e_{i i} \beta \equiv \beta \quad \bmod \mathfrak{P}_{A}^{r}, \quad \beta \in \mathfrak{D}_{s} .
$$

It follows that $\left(D-D^{\prime}\right)$ induces on $\mathfrak{D}_{s}$ a derivation of $\mathfrak{D}_{s}$ into $\mathfrak{D}_{s} / \mathfrak{P}_{s}^{r}$.

On the other hand, for any derivation $D$ of $\mathfrak{D}_{s}$ into $\mathfrak{D}_{s} / \mathfrak{P}_{s}^{r}$, there is uniquely determined derivation $D^{*}$ of $\mathfrak{D}_{A}$ into $\mathfrak{D}_{A} / \mathfrak{P}_{A}^{r}$ such that $D^{*}$ is an extension of $D$ and $D^{*}\left(e_{h t}\right) \equiv 0\left(\mathfrak{P}_{A}^{r}\right)$, namely $D^{*}\left(\sum e_{h t} \alpha_{h t}\right) \equiv \sum e_{h t} D\left(\alpha_{h t}\right)$. This proves our theorem.

Now we consider an algebra $U=\left(\boldsymbol{r}_{0}, \tau, W\right)=\sum_{o} u_{\sigma} W$ such that $u_{\sigma} u_{\text {: }}$ $=r_{\sigma, \tau} u_{\sigma \tau}$, and $u_{\sigma} \alpha u_{\sigma}^{-1}=\alpha^{\sigma}$ for $\alpha \in W$, where $W$ is an unramified Galois extension of $K$ and $r_{\sigma, \tau}$ is a factor set of units in $W$. Moreover we assume that $k \subseteq K$ and the residue class field of $W$ is separable over that of $k$. Then $\mathfrak{D}_{U}$ $=\sum_{\sigma} u_{\sigma} \searrow_{W}$ is a maximal order of $U$ and $U / K$ is unramified. ${ }^{7}$

Let $S=\left(\pi_{0}, Z, \sigma\right)$ considered before. Then the product $\mathfrak{D}_{A}$ of $\mathfrak{D}_{U}$ and $\mathfrak{D}_{s}$ is a maximal order of $A=U \times{ }_{K} S$. We identify $\alpha=\alpha \times 1$ for $\alpha \in U$ and denote $\bar{\beta}=1 \times \beta$ for $\beta \in S$; any element of $\varrho_{A}$ has the form $\sum_{\sigma, h, j, i} u_{\sigma} \omega_{0}^{h} \bar{\pi}^{j} \bar{\omega}^{i} \boldsymbol{a}_{\circ h j i}$, $a_{\supset h j i} \in \mathfrak{D}_{K}$ where $\left(1, \omega_{0}, \omega_{0}^{2}, \ldots\right)$ is a base of $\mathfrak{D}_{W}$ over $\mathfrak{D}_{K}$, further we can assume that $\left(1, \omega_{0}, \omega_{0}^{2}, \ldots\right)$ is an integral base of the maximal inertial subfield of $W$ over $k$. It is known that any simple algebra $\bar{A}$ over $k$ such that the residue class field of $\bar{A}$ is separable over that of $k$, is similar to a $U \times S$, where $U$ and $S$ are algebras such as stated above; ${ }^{7)}$ in this case we can assume that $r_{r, \tau}$ belong to the maximal inertial subfield of $W$ over $k$.

Theorem 4. $H^{1}\left(\mathfrak{D}_{\bar{A}}, \mathfrak{D}_{k} ; \mathfrak{D}_{\bar{A}} / \mathfrak{P}_{A}^{r}\right) \cong H^{1}\left(\mathfrak{D}_{s}, \mathfrak{D}_{k} ; \mathfrak{D}_{S} / \mathfrak{P}_{s}^{r}\right)$, where $\mathfrak{D}_{\bar{A}}$ is a naximal order of $\bar{A}$ and $\mathfrak{P}_{\bar{A}}$ is the two-sided prime ideal of $\mathfrak{D}_{\bar{A}}$.

7) T. Nakayama [6], or O. F. G. Schilling [7], p. 151-156. 
Proof. In virtue of Theorem 3, to prove this it is enongh to prove that $H^{1}\left(\mathfrak{D}_{A}, \mathfrak{D}_{k} ; \mathfrak{D}_{A} / \mathfrak{P}_{A}^{r}\right) \cong H^{1}\left(\mathfrak{D}_{s}, \mathfrak{D}_{k} ; \mathfrak{D}_{S} / \mathfrak{P}_{s}^{r}\right)$. Let $D$ be a derivation in $\mathfrak{D}\left(\mathfrak{D}_{A}, \mathfrak{D}_{k} ;\right.$ $\left.\mathfrak{D}_{A} / \mathfrak{P}_{A}^{r}\right)$, and put

$$
D\left(\omega_{0}\right)=\sum_{\sigma} u_{\sigma} \gamma_{\sigma}, \quad \gamma_{\sigma}=\sum_{h, j, i} \omega_{0}^{h} \bar{\pi}^{j} \bar{\omega}^{i} a_{\sigma h j i}, \quad a_{o h j i} \in \mathfrak{D}_{K},
$$

where we may assume $u_{e}=1$. We put $\sum_{\sigma \neq e} u_{\sigma} \gamma_{\sigma}\left(\omega_{0}-\omega_{0}^{\sigma}\right)^{-1} \equiv t \bmod \mathfrak{P}_{A}^{r}$, since $\omega_{0}-\omega_{0}^{R}$ is not divisible by $\mathfrak{P}_{A}$.

Let $D^{\prime}$ be the inner derivation defined by $D^{\prime}(\alpha)=t \alpha-\alpha t$. Then

$$
\begin{aligned}
D^{\prime}\left(\omega_{0}\right)=t \omega_{0}-\omega_{0} t & \equiv \sum_{\sigma \neq e} u_{\sigma} \gamma_{\sigma}\left(\omega_{0}-\omega_{0}^{\sigma}\right)^{-1} \omega_{\mathrm{i}}-\sum_{\sigma \neq e} \omega_{0} u_{\sigma} \gamma_{\sigma}\left(\omega_{0}-\omega_{0}^{\sigma}\right)^{-1} \\
& \equiv \sum_{\sigma \neq e} u_{\sigma} \gamma_{\sigma}\left(\omega_{0}-\omega_{0}^{\sigma}\right)^{-1} \omega_{0}-\sum_{\sigma \neq e} u_{\sigma} \gamma_{\sigma}\left(\omega_{0}-\omega_{0}^{\sigma}\right)^{-1} \omega_{0}^{\sigma} \equiv \sum_{\sigma \neq e} u_{\sigma} \gamma_{\sigma} .
\end{aligned}
$$

Hence

$$
\left(D-D^{\prime}\right)\left(\omega_{0}\right) \equiv \gamma_{e} \quad \bmod \mathfrak{P}_{A}^{r} .
$$

Now let $T=k\left(\omega_{0}\right)$ and let $F(X)$ be the irreducible polynomial with coefficients in $k$ such that $F\left(\omega_{0}\right)=0$. Then $T / k$ is unramified and we get $F^{\prime}\left(\omega_{0}\right) \neq 0$ $\bmod \mathfrak{P}_{A}$. Since $\left(D-D^{\prime}\right)\left(\omega_{0}\right)$ is commutable with $\omega_{0}$, it holds that

$$
\left(D-D^{\prime}\right)\left(F\left(\omega_{0}\right)\right) \equiv F^{\prime}\left(\omega_{0}\right)\left(D-D^{\prime}\right)\left(\omega_{0}\right) \quad \bmod \mathfrak{P}_{A}^{r} .
$$

Therefore we have

$$
\left(D-D^{\prime}\right)\left(\omega_{0}\right) \equiv 0 \quad\left(\mathfrak{B}^{r}\right) .
$$

Moreover since $\omega_{0}^{\jmath} \in T$ we get

$$
\left(D-D^{\prime}\right)\left(\omega_{0}^{R}\right) \equiv 0 \quad\left(\mathfrak{P}_{A}^{r}\right) .
$$

Therefore we may assume without loss of generality that

$$
D\left(\omega_{0}\right) \equiv 0 \quad\left(\mathfrak{P}_{A}^{r}\right), \quad D\left(\omega_{0}^{\tau}\right) \equiv 0 \quad\left(\mathfrak{P}_{A}^{r}\right) .
$$

Next we put

$$
D\left(u_{3}\right) \equiv \sum_{\tau} u_{\tau} \gamma_{\tau} .
$$

Then since $u_{\sigma} \omega_{0}^{\sigma}=\omega_{0} u_{\sigma}$ and $\omega_{0}^{\tau}$ is commutable with $\gamma_{\tau}$, we have

$$
\begin{aligned}
D\left(u_{o}\right) \omega_{0}^{\sigma}-\omega_{0} D\left(u_{\sigma}\right) & \equiv \sum_{\tau} u_{\tau} \gamma_{\tau} \omega_{0}^{\sigma}-\sum_{\tau} \omega_{0} u_{\tau} \gamma_{\tau} \\
& \equiv \sum_{\tau} u_{\tau} \gamma_{\tau}\left(\omega_{0}^{\sigma}-\omega_{0}^{\tau}\right) \equiv 0 \quad \bmod \mathfrak{P}_{A}^{r} .
\end{aligned}
$$

As we can see from this that

$$
\sum_{\tau \neq \sigma} u_{\tau} \gamma_{\tau} \equiv 0 \quad \bmod \mathfrak{P}_{A}^{r},
$$


we get

$$
D\left(u_{\sigma}\right) \equiv u_{\sigma} \gamma_{o}, \quad \gamma_{\sigma}=\sum_{j, i} \alpha_{\sigma j i} \bar{\pi}^{j} \bar{\omega}^{i}, \quad \alpha_{\sigma j i} \in D_{W} .
$$

Therefore

$$
\begin{aligned}
D\left(u_{\sigma} u_{\tau}\right) & \equiv D\left(u_{\sigma}\right) u_{\tau}+u_{\sigma} D\left(u_{\tau}\right) \equiv u_{\sigma} \sum_{j, i} \alpha_{\sigma j i} \bar{\pi}^{j} \bar{\omega}^{i} u_{\tau}+u_{\sigma} u_{\tau} \sum_{j, i} \alpha_{\tau j i} \bar{\pi}^{j} \bar{\omega}^{i} \\
& \equiv u_{\sigma} u_{\tau} \sum_{j, i} \alpha_{\sigma j i}^{\tau} \bar{\pi}^{j} \bar{\omega}^{i}+u_{\sigma} u_{\tau} \sum_{j, i} \alpha_{\tau j i} \bar{\pi}^{j} \bar{\omega}^{i} .
\end{aligned}
$$

On the other hand, since all $r_{0, \tau}$ belong to the maximal inertial subfield $T$ of $W$ over $k, r_{\sigma, \tau} \in \sum_{i} \bigcirc_{k} \omega_{0}^{i}, D\left(r_{o, \tau}\right) \equiv 0$, so that

$$
D\left(u_{\sigma} u_{\tau}\right)=D\left(r_{\sigma, \tau} u_{\sigma \tau}\right) \equiv r_{\sigma, \tau} u_{\sigma \tau} \sum_{j, i} \alpha_{\sigma \tau j i} \bar{\pi}^{j} \bar{\omega}^{i} \quad \bmod \mathfrak{P}_{A}^{r} .
$$

Therefore we get

$$
r_{\sigma, \tau} u_{\sigma \tau} \sum_{j, i}\left(\alpha_{\sigma j i}^{\tau}+\alpha_{\tau j i}-\alpha_{\sigma \tau j i}\right) \bar{\pi}^{j} \bar{\omega}^{i} \equiv 0 \quad \bmod \left(\mathfrak{P}_{A}^{r}\right)
$$

hence,

$$
\begin{aligned}
& \left(\alpha_{\sigma j i}^{\tau}+\alpha_{\tau j i}-\alpha_{\sigma \tau j i}\right) \bar{\pi}^{j} \bar{\omega}^{i} \equiv 0 \quad \bmod \mathfrak{P}_{A}^{r} \\
& \alpha_{\sigma j i}^{\tau}+\alpha_{\tau j i} \equiv \alpha_{\sigma \tau j i} \quad \bmod \mathfrak{P}_{A}^{r-j} .
\end{aligned}
$$

Therefore, in virtue of Lemma 5 there exist $\beta_{j i}$ in $\mathfrak{D}_{W}$ such that

$$
\begin{aligned}
\alpha_{\sigma j i} & \equiv \beta_{j i}-\beta_{j i}^{\beta} \quad \bmod \mathfrak{P}_{A}^{r-j} \\
\alpha_{\sigma j i} \bar{\pi}^{j} \bar{\omega}^{i} & \equiv \beta_{j i} \bar{\pi}^{j} \bar{\omega}^{i}-\beta_{j i}^{\tilde{\gamma}} \bar{\pi}^{j} \bar{\omega}^{i} \bmod \mathfrak{P}_{A}^{r} \\
\sum_{,, i} \alpha_{\sigma j i} \bar{\pi}^{j} \bar{\omega}^{i} & \equiv \sum_{j, i} \beta_{j i} \bar{\pi}^{j} \bar{\omega}^{i}-\sum_{j, i} \beta_{j i}^{\sigma} \bar{\pi}^{j} \bar{\omega}^{i} \bmod \mathfrak{P}_{A}^{r} . \\
D\left(u_{\sigma}\right) & \equiv u_{\sigma} \sum_{j, i} \alpha_{\sigma j i} \bar{\pi}^{j} \bar{\omega}^{i} \equiv u_{\sigma}\left(\sum_{j, i} \beta_{j i} \bar{\pi}^{j} \bar{\omega}^{i}\right)-\left(\sum_{j, i} \beta_{j i} \bar{\pi}^{j} \bar{\omega}^{i}\right) u_{\sigma} \\
D\left(\omega_{0}\right) & \equiv \omega_{0}\left(\sum \beta_{j i} \bar{\pi}^{j} \bar{\omega}^{i}\right)-\left(\sum \beta_{j i} \bar{\pi}^{j} \bar{\omega}^{i}\right) \omega_{0} \equiv 0 .
\end{aligned}
$$

Therefore by considering the equivalence by inner derivations, we can assume

$$
\begin{array}{ll}
D\left(u_{\sigma}\right) \equiv 0 & \bmod \mathfrak{P}_{A}^{r} \\
D\left(\omega_{0}\right) \equiv 0 & \bmod \mathfrak{P}_{A}^{r} .
\end{array}
$$

Now let $\lambda$ be an element in $\mathfrak{D}_{s}$ and let

$$
D(\bar{\lambda}) \equiv \sum_{\sigma h j i} a_{\sigma h j i} u_{\sigma} \omega_{0}^{h} \bar{\pi}^{j} \bar{\omega}^{i} \quad \bmod \mathfrak{P}_{A}^{r}
$$

Then, since $\bar{\lambda} \omega_{0}=\omega_{0} \bar{\lambda}$

$$
\begin{aligned}
& \sum_{\sigma, h} u_{\sigma} \omega_{0}^{h}\left(\sum_{j, i} a_{\sigma h j i} \bar{\pi}^{j} \bar{\omega}^{i}\right) \omega_{0}-\omega_{0} \sum_{\sigma, h} u_{\sigma} \omega_{0}^{h}\left(\sum_{j, i} a_{\sigma h j i} \bar{\pi}^{j} \bar{\omega}^{i}\right) \equiv 0 \\
& \sum_{\sigma, h} u_{\sigma} \omega_{0}^{h}\left(\omega_{0}-\omega_{0}^{\sigma}\right)\left(\sum_{j, i} a_{\sigma h j i} \bar{\pi}^{j} \bar{\omega}^{i}\right) \equiv 0 \quad\left(\mathfrak{P}_{A}^{r}\right) .
\end{aligned}
$$


From this it follows that

$$
D(\lambda) \equiv \sum_{h} \omega_{0}^{h}\left(\sum_{j, i} a_{h j i} \bar{\pi}^{j} \bar{\omega}^{i}\right) \quad \bmod \mathfrak{P}_{A}^{r} .
$$

Similarly, since $\boldsymbol{u}_{\sigma} \bar{\lambda}=\bar{\lambda} \boldsymbol{u}_{0}$,

As

$$
\begin{aligned}
& \sum_{h} u_{\sigma}\left(\omega_{0}^{h}-\left(\omega_{0}^{h}\right)^{\sigma}\right)\left(\sum_{j, i} a_{h j i} \bar{\pi}^{j} \bar{\omega}^{i}\right) \equiv 0 \\
& \sum_{j, i}\left(\sum_{h}\left(\omega_{0}^{h}-\left(\omega_{0}^{h}\right)^{\sigma}\right) a_{h j i}\right) \bar{\pi}^{j} \bar{\omega}^{i} \equiv 0 \\
& \sum_{h \neq 0}\left(\omega_{0}^{h}-\left(\omega_{0}^{h}\right)^{\sigma}\right) a_{h j i} \equiv 0\left(\mathfrak{P}_{A}^{r-j}\right) .
\end{aligned}
$$

$$
\begin{aligned}
& \omega_{0}-\omega_{0}^{o}, \omega_{0}^{2}-\left(\omega_{0}^{\sigma}\right)^{2}, \ldots, \omega_{0}^{n-1}-\left(\omega_{0}^{\sigma}\right)^{n-1} \\
& \omega_{0}-\omega_{0}^{\tau}, \omega_{0}^{2}-\left(\omega_{0}^{\tau}\right)^{2}, \ldots, \omega_{0}^{n-1}-\left(\omega_{0}^{\tau}\right)^{n-1} \neq 0 \\
& \begin{array}{ccccccccc}
\cdot & \cdot & \cdot & \cdot & \cdot & \cdot & \cdot & \cdot & \cdot \\
\cdot & \cdot & \cdot & \cdot & \cdot & \cdot & \cdot & \cdot &
\end{array}
\end{aligned}
$$

we see that

$$
a_{h j i} \equiv 0 \quad \bmod \mathfrak{P}_{A}^{r-j} \quad(h \neq 0)
$$

Therefore

$$
D(\lambda) \equiv \sum_{j, i} a_{0 j i} \bar{\pi}^{j} \bar{\omega}^{i} \quad \bmod \mathfrak{P}_{A}^{r}
$$

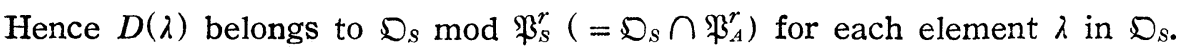

Conversely for any derivation $D$ in $\mathfrak{D}\left(\mathfrak{D}_{s}, \mathfrak{D}_{k} ; \mathfrak{D}_{s} / \mathfrak{P}_{s}^{r}\right)$, there exists a derivation in $\mathfrak{D}\left(\mathfrak{D}_{A}, \mathfrak{D}_{k} ; \mathfrak{D}_{A} / \mathfrak{F}_{A}^{r}\right)$ which is uniquely determined extension of $D$ satisfying (1); therefore our theorem is proved.

Let $\bar{A}$ be a simple algebra over a complete field with respect to a valuation, considered above. The length of composition series of $H^{1}\left(\mathfrak{D}_{\bar{A}}, \mathfrak{D}_{k}\right.$; $\left.\mathfrak{D}_{\bar{A}} / \mathfrak{P}_{\bar{A}}^{r}\right)$ as $\mathfrak{D}_{K}$-module shall be called the dimension of $H^{1}\left(\mathfrak{D}_{\bar{A}}, \mathfrak{D}_{k} ; \mathfrak{D}_{\bar{A}} / \mathfrak{P}_{\bar{A}}^{r}\right)^{\left.{ }^{8}\right)}$

THEOREM 5. Let $\mathfrak{P}_{K}^{d}$ denote the different of $K$ with respect to $k$. Then the maximal dimension of $H^{1}\left(\mathfrak{D}_{\bar{A}}, \mathfrak{D}_{k} ; \mathfrak{D}_{\bar{A}} / \mathfrak{P}_{\bar{A}}^{r}\right)$ is

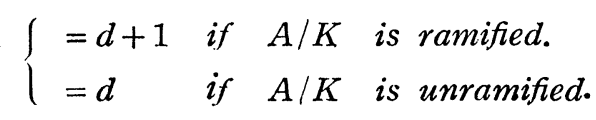

The largest two-sided ideal $\overline{\mathfrak{D}}(\bar{A} / k)$ such that $H^{1}\left(\mathfrak{D}_{\bar{A}}, \mathfrak{D}_{k} ; \overline{\mathfrak{D}}(\bar{A} / k)\right)$ gives the maximal dimension is

8) M. Moriya [4]. 


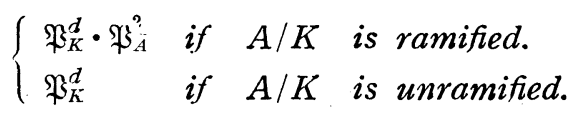

Proof. It is known that the maximal dimension of $\mathscr{D}\left(\mathfrak{D}_{K}, \mathfrak{D}_{k}, \mathfrak{D}_{K} / \mathfrak{D}_{K}\right.$ $\left.\cap \mathfrak{P}_{K}^{r}\right)$ is $d .^{9)}$ Our theorem follows immediately from Theorems $1,2,3$ and 4.

Remark. $\bar{D}(\bar{A} / k)$ is also characterized by the property that $H^{1}\left(\mathfrak{D}_{\bar{A}}, \mathfrak{D}_{k} ;\right.$ $\bar{D}(\bar{A} / k))$ gives the maximal dimension as $\mathfrak{D}_{k}$-module.

3. Let $A$ be a simple algebra over a field $k$ which is the quotient field of a Dedekind ring $\mathfrak{D}$ and $\mathfrak{D}_{A}$ a maximal order of $A$ with respect to $\mathfrak{D}$. Let $\mathfrak{A}$ be a two-sided ideal of $\mathfrak{D}_{A}$. Then it is known that $\mathfrak{A}$ is a product of prime ideals $\mathfrak{A}=\Pi \mathfrak{P}_{i}^{m_{i}}$, and $\mathfrak{D}_{A} / \mathfrak{A} \cong \sum_{i} \oplus \mathfrak{D}_{A} / \mathfrak{P}_{i}^{m_{i}}$. Let $K$ be the center of $A$ and let $\mathfrak{D}_{K}$ be the ring of all integral elements of $K$ and $\mathfrak{D}_{k}=\mathfrak{D}$. Let $A_{\mathfrak{P}}$ be the $\mathfrak{P}$ adic extension of $A$ and let $\mathfrak{D}_{\mathfrak{P}}, \overline{\mathfrak{P}}, \mathfrak{A}_{\mathfrak{P}}$ be the $\mathfrak{P}$-adic extension of $\mathfrak{D}_{A}, \mathfrak{P}$ and $\mathfrak{U}$ respectively. We assume that $\mathfrak{D}_{A} / \mathfrak{P}$ is separable over $\mathfrak{D}_{k} / \mathfrak{p}_{0}\left(\mathfrak{p}_{0}=\mathfrak{P} \cap \mathfrak{D}_{k}\right)$ for any $\mathfrak{P}$.

Lemma 7. $H^{1}\left(\mathfrak{D}_{A}, \mathfrak{D}_{k} ; \mathfrak{D}_{A} / \mathfrak{A}\right) \cong \sum_{i} \oplus H^{1}\left(\mathfrak{D}_{A}, \mathfrak{D}_{k} ; \mathfrak{D}_{A} / \mathfrak{P}_{i}^{m_{i}}\right)$

Proof. Since $\mathfrak{D}_{A} / \mathfrak{A} \cong \sum_{i} \oplus \mathfrak{D}_{A} / \mathfrak{P}_{i}^{m_{i}}$, we can prove easily this lemma.

Lemma 8. $\left.H^{1}\left(\mathfrak{D}_{A}, \mathfrak{D}_{k} ; \mathfrak{D}_{A} / \mathfrak{H}\right) \cong \sum_{i} \oplus H^{1} \mathfrak{D}_{A}, \mathfrak{D}_{k} ; \mathfrak{D}_{A} / \mathfrak{P}_{i}^{m_{i}}\right)$, and the dimension of $H^{1}\left(\mathfrak{D}_{A}, \mathfrak{D}_{k} ; \mathfrak{D}_{A} / \mathfrak{P}_{i}^{m_{i}}\right)$ as $\mathfrak{D}_{K}$-module is equal to the dimension of $H^{1}\left(\mathfrak{D}_{\mathfrak{P}_{i}}, \mathfrak{D}_{\mathfrak{p}_{0 i}} ; \mathfrak{D}_{\mathfrak{P}_{i}} / \overline{\mathfrak{P}}_{i}^{m}\right)$ as $\mathfrak{D}_{\mathfrak{p}_{i}}$-module, where $\mathfrak{D}_{\mathfrak{p}_{0 i}}$ and $\mathfrak{D}_{\mathfrak{p}_{i}}$ are the $\mathfrak{p}_{0 i}$-adic extens on of $\mathfrak{D}_{k}$ and the $\mathfrak{p}_{i}$-adic extension of $\mathfrak{D}_{K}\left(\mathfrak{p}_{i}=\mathfrak{P}_{i} \cap \mathfrak{D}_{K}\right)$.

Proof. By the preceding lemma $H^{1}\left(\mathfrak{D}_{A}, \mathfrak{D}_{k} ; \mathfrak{D}_{A} / \mathfrak{A}\right) \cong \sum_{i} \oplus H^{1}\left(\mathfrak{D}_{A}, \mathfrak{D}_{k}\right.$; $\left.\mathfrak{D}_{A} / \mathfrak{P}_{i}^{m_{i}}\right)$, moreover we can prove that

$$
\left.H^{1}\left(\mathfrak{D}_{A}, \mathfrak{D}_{k} ; \mathfrak{D}_{\mathfrak{P}} / \overline{\mathfrak{P}}^{m}\right) \cong H^{1}\left(\mathfrak{D}_{\mathfrak{P}}, \mathfrak{D}_{\mathfrak{p}_{\mathfrak{v}}} ; \mathfrak{D}_{\mathfrak{P}} / \overline{\mathfrak{P}}^{m}\right) \quad \text { (as } \mathfrak{D}_{\mathfrak{P}} \text {-module }\right) .
$$

For, let $D \in \mathscr{D}\left(\mathfrak{D}_{\mathfrak{P}}, \mathfrak{D}_{\mathfrak{p}_{0}} ; \mathfrak{D}_{\mathfrak{B}} / \overline{\mathfrak{P}}^{m}\right)$ and let $\alpha \in \mathfrak{D}_{\mathfrak{B}}$. Then $\alpha$ can be written by the form

$$
\alpha=\beta+\pi_{0}^{t} \gamma, \quad \beta \in \mathfrak{D}_{A}, \quad \gamma \in \mathbb{D}_{\Re}, \quad \pi_{0} \text { is a prime element in } \mathfrak{D}_{k} .
$$

Then for sufficiently large $t$

$$
D\left(\pi_{0}^{t} r\right) \equiv 0 \quad \bmod \overline{\mathfrak{P}}^{m} .
$$

9) M. Moriya [4], p. 134, Satz 5. 
Therefore $D$ is determined by the restriction of $D$ on $\mathfrak{D}_{A}$.

Further we can easily see that the length of composition series of $H^{1}\left(\mathfrak{D}_{A}, \mathfrak{D}_{k} ; \mathfrak{D}_{\mathfrak{P}} / \overline{\mathfrak{P}}^{m}\right)$ as $\mathfrak{D}_{\mathfrak{p}}$-module is equal to that of $H^{1}\left(\mathfrak{D}_{A}, \mathfrak{D}_{k} ; \mathfrak{D}_{A} / \mathfrak{P}^{m}\right)$ as $\mathfrak{D}_{K}$-module.

Let $\overline{\mathfrak{D}}=\overline{\mathfrak{D}}(A / k)$ be the largest two-sided ideal of $\mathfrak{D}_{A}$, such that $H^{1}\left(\mathfrak{O}_{K}, \mathfrak{D}_{k}\right.$; $\left.\mathfrak{D}_{A} / \mathcal{D}\right)$ gives the maximal dimension (as $\mathfrak{D}_{K}$-module). Then $H^{1}\left(\mathfrak{D}_{A}, \mathfrak{D}_{k} ; \mathfrak{D}_{A} / \mathfrak{D}\right.$ ) also gives the maximal dimension as $\mathfrak{D}_{k}$-module.

THEOREM 6. The $\mathfrak{P}$-contribution of $\bar{D}$ is $\mathfrak{P}^{\varepsilon} \mathfrak{p}^{d}$, where $\mathfrak{p}^{d}$ is the $\mathfrak{p}$-contribution of the different of $K / k$, and

$$
\varepsilon=\left\{\begin{array}{l}
0 \text { when } \mathfrak{P} \text { does not ramify over } K . \\
2 \text { when } \mathfrak{P} \text { ramifies over } K .
\end{array}\right.
$$

Proof. From Theorem 5 and Lemma 8 we can prove easily.

Corollary. $\mathfrak{P}$ devides $\overline{\mathfrak{D}}(A / k)$ if and only if $\mathfrak{B}$ ramifies over $k$. Moreover, let $L$ be any subfield of the center of $A$. Then $\bar{D}(A / k)=\bar{D}(\mathrm{~A} / L) \bar{D}(L / k)$.

Let $B$ be a semi-simple algebra over $k$. Then $B$ is the direct sum of simple algebras $A_{i}$ and we see easily that each maximal order $D$ of $B$ is the direct sum of maximal orders $\mathfrak{D}_{i}$ of $A_{i}$. Moreover every ideal $\mathfrak{A}$ of $\mathfrak{D}$ is the direct sum of ideals $\mathfrak{A}_{i}$ of $\mathfrak{D}_{i}$, and a prime ideal of $\mathfrak{D}$ is the direct sum of a prime ideal $\overline{\mathfrak{P}}_{i}$ of $\mathfrak{D}_{i}$ and $\mathfrak{D}_{j}(j \neq i)$, denoted by $\mathfrak{P}_{i}$. Let $\mathfrak{U}=\Pi \mathfrak{P}_{i}^{m_{i}}$. Then $H^{1}\left(\mathfrak{D}, \mathfrak{D}_{k} ; \mathfrak{D} / \mathfrak{H}\right) \cong \sum_{i} \oplus H^{1}\left(\mathfrak{D}, \mathfrak{D}_{k} ; \mathfrak{D} / \mathfrak{P}_{i}^{m_{i}}\right), H^{1}\left(\mathfrak{D}, \mathfrak{D}_{k} ; \mathfrak{D} / \mathfrak{P}_{i}^{m_{i}}\right) \cong \sum_{i} \oplus H^{1}\left(\mathfrak{D}_{j}\right.$, $\left.\mathfrak{D}_{k} ; \mathfrak{D}_{i} / \mathfrak{P}_{i}^{m_{i}}\right) \cong H^{1}\left(\mathfrak{D}_{i}, \mathfrak{D}_{k} ; \mathfrak{D}_{i} / \overline{\mathfrak{P}}_{i}^{m_{i}}\right)$; hence $H^{1}\left(\mathfrak{D}, \mathfrak{D}_{k} ; \mathfrak{D} / \mathfrak{A}\right) \cong \sum_{i} \oplus H^{1}\left(\mathfrak{D}_{i} / \mathfrak{D}_{k} ;\right.$ $\left.\mathfrak{D}_{i} / \overline{\mathfrak{P}}_{i}^{m_{i}}\right)$. Therefore if $\overline{\mathfrak{D}}=\overline{\mathfrak{D}}(B / k)$ denotes the largest two sided ideal of $\mathfrak{D}$, such that $H^{1}\left(\mathfrak{D}, \mathfrak{D}_{k} ; \mathfrak{D} / \bar{D}\right)$ gives the maximal dimension, then the same properties as Theorem 6 and its corollary also hold for $\bar{D}(B / k)$.

\section{REFERENCES}

[1] A. Weil, Differentiation in algebraic number fields, Bull. Amer. Math. Soc., Vol. 49 (1943), p. 41.

[2] Y. Kawada, On the derivations in number fields, Ann. of Math., Vol. 54 (1951), p. 302314.

[3] Y. Kawada, On the Derivations in Simple Algebras, Scientific Papers of the Colledge of Ceneral Education University of Tokyo, Vol. II (1952), p. 1-8.

[4] M. Moriya, Theorie der Derivationen und Körperdifferenten, Mathematical Journal of Okayama University, Vol. 2 (1952-53), p. 111-148. 
[5] H. Hasse, Über p-adische Schiefkörper und ihre Bedeutung für die Arithmetik hyperkomplexen Zahlsysteme, Math. Ann., Bd. 104, p. 495-534.

[6] T. Nakayama, Divisionsalgebren über diskret bewerteten perfekten Körpern, Crelle 178, p. 11-13.

[7] O. F. G. Schilling, The Theory of valuations, New York (1950).

Mathematical Institute, Nagoya University 